\title{
A 29-year-old pregnant woman with a history of anthracycline-induced clinical heart failure
}

\author{
Valentina Scheggi*, Fabio Mori \\ Department of Cardiology, Azienda Ospedaliero-Universitaria Careggi, Firenze, Italy; \\ *Corresponding Author: valentina.scheggi@libero.it
}

Received 11 November 2010; revised 2 November 2010; accepted 8 November 2011

\begin{abstract}
The number of women with heart disease who reach childbearing age in a good functional state increases continuously as advances in diagnosis and treatment improve overall health and prognosis. The cardiologist's role is to give the woman an estimate of both maternal and fetal risk to allow her to make an informed decision about embarking on a pregnancy, and to provide appropriate antenatal care. There are only a few data about the natural history of anthracycline-induced cardiomyopathy during preg- nancy; we report our experience of a 29-year- old pregnant woman with a history of anthracycline-induced clinical heart failure.
\end{abstract}

Keywords: Anthracyclines; Heart Failure; Pregnancy; Cardiomyopathy

\section{INTRODUCTION}

Heart disease is present in $0.5-1 \%$ of all pregnancies and accounts for about $10-15 \%$ of all maternal death. Management of these patients requires teamwork of obstetricians, neonatologists, cardiologists, anesthetists and sometimes cardiac surgeons but there are only a few data in the literature to guide clinicians in maternal and fetal care. We report the case of a pregnant woman with anthracycline-induced cardiomyopathy and a review of the literature.

\section{METHODS}

We evaluated a 29-year-old woman in the 5th week of pregnancy. During childhood she had osteosarcoma treated with anthracyclines and at the age of 25 years she presented anthracycline-induced NYHA class IV heart failure with markedly reduced systolic left ventricular function (EF 24\%) and severe mitral regurgitation. She was treated with ACE-inhibitors, beta-blockers and diuretics with good clinical result and improving systolic left ventricular function with EF until $47 \%$ and trivial mitral regurgitation.

At the time of evaluation the pulse was $83 \mathrm{bpm}$, blood pressure was $90 / 60 \mathrm{mmHg}$, without signs of heart failure; the electrocardiogram was normal and p-BPN was 600 $\mathrm{pg} / \mathrm{ml}$.

The ACE-inhibitor was discontinued and she was treated with bisoprolol and LMWH.

Her clinical conditions and left systolic ventricular function continued to be stable and she vaginally delivered a healthy child at 35 weeks of pregnancy.

The follow up after delivery was uneventful with stable EF.

\section{DISCUSSION}

Mortality among minimally symptomatic pregnant women with cardiac disease is about $1 \%$, as among the healthy general population. In contrast, severely symptomatic women have been reported to experience a mortality risk up to $5-15 \%$.

Just a small number of parameters allow dichotomic classification into high-risk and low-risk patients.

In a prospective multicentre study enrolling 562 women, Siu et al. [1] identified poor functional NYHA class or cyanosis, left ventricular systolic dysfunction ( $\mathrm{EF}<50 \%$ ), and left heart obstruction as major determinants for maternal cardiac complications.

Neonatal complications (20\% of pregnancies) were associated with poor functional class or cyanosis, left heart obstruction, anticoagulation, smoking, and multiple gestations.

Verena Stangl et al. [2] found that women at high-risk (as defined above) had a 6.1-fold higher maternal complication rate and a 6.1 times higher fetal/neonatal event rate; $64.7 \%$ of the high-risk women delivered prematurely, compared to $16.4 \%$ in the low-risk group. 
In low-risk women, fetal complications were comparable to those reported for the general population but preterm birth rate was slightly higher.

Thorne et al. [3] showed that the risk of maternal death is approximately 7\% if the patient is in New York Heart Association (NYHA) functional class III or IV. Other adverse risk factors include ejection fraction < $20 \%$, mitral regurgitation, right ventricular failure, atrial fibrillation, and systemic hypotension.

The natural history of specific types of cardiomyopathy during pregnancy is unknown and there are only a few data about pregnant women with anthracycline-induced cardiomyopathy.

Anthracycline-cardiotoxicity can become manifest as either clinical heart failure or asymptomatic cardiac dysfunction. Both can develop also years after the cessation of treatment, as happened in our patient.

Elvira C. van Dalen [4] evaluated the incidence of peripartum anthracycline-induced clinical heart failure in a cohort of 53 women. This study demonstrates a low risk in childhood cancer survivors.

It is worth noticing that 2 of the 53 women included in this study developed heart failure shortly after the end of anthracycline therapy and that neither of them developed any peripartum cardiac problems.

About therapy, sodium and physical activity restrictions, in association with drugs like digoxin and furosemide, help control heart failure during pregnancy.

Hydralazine, with or without nitrates, is an alternative to angiotensin-converting enzyme inhibitors, that are associated with side effects.

As anti-arrhythmics, amiodarone may be toxic but beta-blockers can be used safely.

Finally, patients with ventricular dysfunction must be anticoagulated with heparin at prophylactic doses to prevent thromboembolism.

\section{CONCLUSION}

In summary, pregnancy outcome in women who received anthracyclines for malignancy in childhood is generally favorable. Those with left ventricular dysfunction, as our patient, should be considered at increased risk but probably the most important prognostic factor is the NYHA class.

\section{REFERENCES}

[1] Siu, S.C., Sermer, M., Colman, J.M., Alvarez, A.N., Mercier, L.-A., Morton, B.C., Kells, C.M., Bergin, M.L., Kiess, M.C., Marcotte, F., Taylor, D.A., Gordon, E.P., Spears, J.C., Tam, J.W., Amankwah, K.S., Smallhorn, J.F., Farine, D. and Sorensen, S. (2001) Prospective multicenter study of pregnancy outcomes in women with heart disease. Circulation, 104, 515-521. doi:10.1161/hc3001.093437

[2] Stangl, V., Schad, J., Gossing, G., Borges, A., Baumann, G., Stangl, K. (2008) Maternal heart disease and pregnancy outcome: A single-centre experience. European Journal of Heart Failure, 10, 855-860. doi:10.1016/j.ejheart.2008.07.017

[3] Thorne, S.A. (2004) Pregnancy in heart disease. Heart, 90, 450-456. doi:10.1136/hrt.2003.027888

[4] Dalen, E.C.V, Pal, H.J.H.V.D., Bos, C.V.D., Kok, W.E.M., Caron, H.N. and Kremer, L.C.M.. Clinical heart failure during pregnancy and delivery in a cohort of female childhood cancer survivors treated with anthracyclines (2006) European Journal of Cancer, 42, 25492553. doi:10.1016/j.ejca.2006.04.014 